\title{
EDITORIAL \\ Flow diverters: one device does not fit all
}

\author{
R. Loch Macdonald, MD, PhD \\ Division of Neurosurgery, St. Michael's Hospital, Labatt Family Centre of Excellence in Brain Injury and Trauma Research, \\ Keenan Research Centre for Biomedical Science, and the Li Ka Shing Knowledge Institute of St. Michael's Hospital, \\ Department of Surgery, University of Toronto, Ontario, Canada
}

$\mathrm{O}$ $\mathrm{NE}$ of the few groups investigating intracranial aneurysm hemodynamics reports in silico modeling of changes in computational fluid dynamics (CFD) of cerebral aneurysms after treatment with flowdiverting stents. ${ }^{7}$ The authors describe 3 patients with intracranial aneurysms treated with flow-diverting Pipeline Embolization Devices (PEDs). They calculated aneurysm flow velocity, inflow rate, wall shear stress ([WSS]; a measure of change in flow into the aneurysm) and turnover time (a measure of stasis in the aneurysm) before and after placement of the PED, and they correlate this with whether or not the aneurysm became occluded over time

The PED, along with the SILK and SURPASS flow diverters (FDs), are intracranial stents made of platinum and cobalt chromium wires (PED and SURPASS) or nickeltitanium alloy (nitinol-SILK) that cover about $30 \%$ of the inside of the artery in which they are deployed. For comparison, stents like the Neuroform stent cover less than $10 \%$ of the artery wall. ${ }^{2}$ The PED was approved by the US FDA in April 2011. This was based on data from 131 patients studied between 2007 and 2009.,5 The indication for use was for large or giant, wide-necked aneurysms of the internal carotid artery (ICA) in patients older than 22 years. Use has expanded widely beyond this, with spectacular results in some of these off-label indications. ${ }^{4}$ These FDs are probably going to be only the first generation of technology to occlude cerebral aneurysms without having to enter the aneurysm sac. Repairing intracranial aneurysms with FDs, while often highly effective in cases that are difficult to treat by endovascular coiling or surgery, carries risks and may fail to occlude the aneurysm. One can envision numerous advancements to overcome limitations like the requirement for dual antiplatelet therapy, concerns about treating bifurcation aneurysms, limited endovascular options if flow diversion fails because the aneurysm cannot be entered, and risks of bleeding in treatment of ruptured aneurysms.
It is the mechanisms of occlusion that are addressed in the paper by Xiang et al. ${ }^{7}$ They describe 3 very different cases, and it is not clear how these were selected and why only 3 are reported. The first patient had a fusiform vertebral artery aneurysm treated with PED, and the aneurysm occluded in 3 months. The posterior inferior cerebellar artery (PICA) arose from the distal part of the aneurysm and it remained patent. The second case was a large supraclinoid ICA aneurysm that also occluded 6 months after PED placement. The third patient had 2 ICA aneurysms. One occluded in 6 months and the other remained patent at 9 months. Patency was suspected to be due to outflow from the carotid artery into the adjacent ophthalmic artery (OphA). In the first case, however, a large adjacent artery (the PICA) was not a source of the failure of the aneurysm to regress. Furthermore, on the images published, it looks like the OphA was in proximity to the aneurysm in the second case, even though this aneurysm regressed.

Thus, the first point is that there are factors other than the CFD parameters that affect whether FDs work. This is highlighted by the in silico modeling shown in Fig. 7. Basically, in all cases after PED placement there was a reduction in aneurysm flow velocity, inflow, and WSS (surrogate for flow) and an increase in turnover time (meaning more stasis). But as noted above, this doesn't correlate with successful aneurysm occlusion. This highlights what we know-that these are very different aneurysms. One is a fusiform dilation in the subarachnoid space of an elderly person, another is a complex set of 2 familial saccular aneurysms, one of which is probably extradural and covered in bone or dura mater, or at least not CSF. The pathogenesis, pathology, and CFD of a fusiform aneurysm must be very different from a saccular aneurysm.

There have been other efforts to model CFD before and after FD treatment. Kulcsár and colleagues modeled CFD before and after treatment of 8 ICA aneurysms with the SILK device. ${ }^{3}$ Their results are similar, with flow di- 
version producing reduced flow velocity and WSS into the aneurysms. They also found 1 aneurysm that did not occlude during follow-up, and it did not have distinctive changes in CFD compared to the ones that did occlude.

The CFD modeling is subject to numerous assumptions, and the methods used are debated in the literature. ${ }^{6}$ The models used here are based on steady state, incompressible, rigid wall, and Newtonian fluid. Patient-specific hemodynamic values (or in CFD terminology, patientspecific inlet flow boundary conditions) were not used. Opinion varies as to how much this matters to the CFD values. The average WSS over the whole inner surface of the aneurysm was used, as well as the overall average of aneurysm velocity, as opposed to changes in flow patterns and such. Another factor is biology and external environment (smoking, platelet and endothelial cell function, response to antiplatelet drugs, and so on). The devices are not customized to the aneurysm. Somehow these factors interact and lead to the limitations shown here. In some cases branch arteries don't affect occlusion, but in others they do.

One can envision important use of this sort of modeling in the future to guide placement of endovascular devices for treatment of aneurysms and other neurovascular conditions, and maybe even to customize devices for the individual aneurysm. Being able to model what will happen in terms of CFD in the aneurysm after it is treated by various methods could be useful. There are limitations at this point though. The results of this paper show that understanding of what makes aneurysms occlude after coiling or flow diversion is incomplete. The authors examine changes in various CFD parameters but it is not known if these are the key ones that mediate aneurysm occlusion, whether the changes are associative in nature and not causal, and if there are other factors that mediate aneurysm growth or regression. The authors astutely discuss the fact that other considerations, such as aneurysm morphology and related factors, as well as treatment-related and individual patient biology must be factored in to the calculation of the efficacy of FDs. The conclusion is the realistic statement that this work confirms that reduction of flow into the aneurysm is associated with a higher chance of occlusion, but that other factors are involved. This is an important first step in development of what will certainly be increasingly sophisticated computer modeling of aneurysms and how to make devices to occlude them. The complexities of intracranial aneurysms suggest that the future will bring (and will require the ability to make) specific devices to treat individual aneurysms.

http://thejns.org/doi/abs/10.3171/2014.12.JNS142537

\section{References}

1. Becske T, Kallmes DF, Saatci I, McDougall CG, Szikora I, Lanzino $\mathrm{G}$, et al: Pipeline for uncoilable or failed aneurysms: results from a multicenter clinical trial. Radiology 267:858868,2013

2. Fiorella D, Woo HH, Albuquerque FC, Nelson PK: Definitive reconstruction of circumferential, fusiform intracranial aneurysms with the pipeline embolization device. Neurosurgery 62:1115-1121, 2008

3. Kulcsár Z, Augsburger L, Reymond P, Pereira VM, Hirsch S,
Mallik AS, et al: Flow diversion treatment: intra-aneurismal blood flow velocity and WSS reduction are parameters to predict aneurysm thrombosis. Acta Neurochir (Wien) 154:1827-1834, 2012

4. Meckel S, McAuliffe W, Fiorella D, Taschner CA, Phatouros C, Phillips TJ, et al: Endovascular treatment of complex aneurysms at the vertebrobasilar junction with flow-diverting stents: initial experience. Neurosurgery 73:386-394, 2013

5. Nelson PK, Lylyk P, Szikora I, Wetzel SG, Wanke I, Fiorella $\mathrm{D}$ : The pipeline embolization device for the intracranial treatment of aneurysms trial. AJNR Am J Neuroradiol 32:34-40, 2011

6. Steinman DA: Computational modeling and flow diverters: a teaching moment. AJNR Am J Neuroradiol 32:981-983, 2011

7. Xiang J, Damiano RJ, Lin N, Snyder KV, Siddiqui AH, Levy EI, et al: High-fidelity virtual stenting: modeling of flow diverter deployment for hemodynamic characterization of complex intracranial aneurysms. J Neurosurg [epub ahead of print June 19, 2015. DOI: 10.3171/2014.11.JNS14497]

\section{Response}

\author{
Jianping Xiang, PhD, ${ }^{1-3}$ Robert J. Damiano, MS, ${ }^{1,3}$ \\ Ning Lin, MD, 2,6 Kenneth V. Snyder, MD, PhD, 1,2,5 \\ Elad I. Levy, MD, 1,2,5 Adnan H. Siddiqui, MD PhD, ${ }^{1,2,5}$ and \\ Hui Meng, $\mathrm{PhD}^{1-4}$
}

${ }^{1}$ Toshiba Stroke and Vascular Research Center, ${ }^{2}$ Department of Neurosurgery, ${ }^{3}$ Department of Mechanical and Aerospace Engineering, ${ }^{4}$ Department of Biomedical Engineering, and ${ }^{5}$ Department of Radiology, University at Buffalo, The State University of New York, Buffalo; and ${ }^{6}$ Department of Neurosurgery, Weill Cornell Medical Center and NewYork-Presbyterian Hospital, New York, New York

We appreciate Dr. Macdonald's thoughtful comments on our paper. As Dr. Macdonald pointed out, we were trying to explore the mechanisms of occlusion of intracranial aneurysms by FD treatment in the current study. As an exploratory study, we randomly chose 3 very different and complex cases, which were good candidates for FD treatment. Even though these 3 cases are very different, it is interesting that we demonstrated the general trend that large flow reduction was correlated with short occlusion time from Aneurysm I, Aneurysm II (treated with 2 PEDs), and Aneurysm III-a in Case 3. Furthermore, from the unexpected result of Aneurysm III-b in Case 3, we also concluded that other factors, such as vascular anatomy, should be considered in the efficacy of FDs. Specifically, we believe that the failure of delayed occlusion of Aneurysm III-b in Case 3 is most likely to be reflective of poor wall apposition of the FD around the carotid siphon with persistent flow outside the FD and into the aneurysm (endoleak). We have started to note the inadequacy of careful, routine, $2 \mathrm{D}$ subtraction angiography to demonstrate this malapposition, and therefore have started to use rotational diluted-contrast 3D CT-like angiography (Low Contrast Imaging, Toshiba; similar to Expert CT by Phillips and Dyna CT by Siemens) to confirm optimal wall apposition by FD. Therefore, the ophthalmic outflow may be an epiphenomenon in this case as in many others.

Our study is only an exploratory study. The main purpose of this study was to demonstrate that the high-fidelity virtual stenting (HiFiVS) modeling of FD deployment 
enables quantitative hemodynamic characterization of complex intracranial aneurysm cases. Through a series of earlier studies, we have developed this finite-element method-based HiFiVS technique ${ }^{2,3}$ to simulate in silico the mechanical process of implanting FDs and we have investigated hemodynamic modifications by different FD delivery strategies. ${ }^{4,6}$ However, the cases used in these virtual experiments were not treated by FDs in real life. In this proof-of-concept study, we thus applied our novel computational device modeling HiFiVS workflow to investigate the flow changes of real patient-specific aneurysms treated by FDs, and we examined whether there was any correlation between their clinical outcome and the amount of hemodynamic changes induced by FDs.

There are only a few reports on the correlation between hemodynamic modifications and occlusion outcome in lesions treated by FDs. Through analysis of 8 ICA aneurysms treated by SILK FDs, Kulcsár et al. ${ }^{1}$ demonstrated the general trend of flow reduction with aneurysm occlusion. Mut et al. ${ }^{5}$ analyzed 23 aneurysms treated by FDs, and also discovered the same trend-that lower posttreatment aneurysmal flow velocity and inflow rate correlated with faster aneurysm occlusion. In this study, as Dr. Macdonald pointed out, even though these 3 aneurysms are very different lesions, we still demonstrated a similar trend. These 3 studies ${ }^{1,5}$ provide the confidence and promise that flow changes by the FDs should play a role in predicting the occlusion outcome and rate. Our preliminary study further revealed that other factors are also important for prediction of treatment outcome. Thus the next logical step would be to investigate hemodynamics as well as other factors (clinical, morphological, and so on) in large cohorts of aneurysm cases treated by FDs and to build prediction models for occlusion outcome (e.g., survival curve models). As implied by Dr. Macdonald, it is worthwhile to continue this effort using the same type of aneurysms (e.g., in the same location).

We envision that in the future we could plan the individualized treatment for patients with aneurysms. After obtaining treatment outcome prediction models from large cohort studies, we could optimize different treatment strategies such as 1 uniform FD, 1 FD with dense compaction at the aneurysm orifice, 2 overlapping FDs, 1 FD with adjunctive coils, or stent-assisted coiling prior to the real treatment. Because FD deployment is a highly variable procedure, an advanced modeling method is needed to realistically capture this process. The HiFiVS technique has the unique capability to recapitulate the entire mechanical and maneuvering processes of FD deployment. Furthermore, this technique has been rigorously validated by in vitro experiments. ${ }^{3}$ This method could serve as a virtual tool to reproduce clinically realistic delivery strategies in silico and enable accurate hemodynamic simulation via CFD a priori for treatment planning, or a posteriori for understanding treatment outcome. We totally agree with Dr. Macdonald that the complexities and heterogeneities of intracranial aneurysms necessitate specific devices to treat individual aneurysms in the future.

Dr. Macdonald also has a concern about the assumptions made when conducting CFD simulation for FDs. As he pointed out, patient-specific inlet flow boundary conditions were not used. We think this assumption should have minimal effect because we only compared the relative hemodynamics after FD treatment instead of absolute values. However, all the assumptions should be evaluated before investigating a large number of cases.

\section{References}

1. Kulcsár Z, Augsburger L, Reymond P, Pereira VM, Hirsch S, Mallik AS, et al: Flow diversion treatment: intra-aneurismal blood flow velocity and WSS reduction are parameters to predict aneurysm thrombosis. Acta Neurochir (Wien) 154:1827-1834, 2012

2. Ma D, Dargush GF, Natarajan SK, Levy EI, Siddiqui AH, Meng H: Computer modeling of deployment and mechanical expansion of neurovascular flow diverter in patient-specific intracranial aneurysms. J Biomech 45:2256-2263, 2012

3. Ma D, Dumont TM, Kosukegawa H, Ohta M, Yang X, Siddiqui $\mathrm{AH}$, et al: High fidelity virtual stenting (HiFiVS) for intracranial aneurysm flow diversion: in vitro and in silico. Ann Biomed Eng 41:2143-2156, 2013

4. Ma D, Xiang J, Choi H, Dumont TM, Natarajan SK, Siddiqui $\mathrm{AH}$, et al: Enhanced aneurysmal flow diversion using a dynamic push-pull technique: an experimental and modeling study. AJNR Am J Neuroradiol 35:1779-1785, 2014

5. Mut F, Raschi M, Scrivano E, Bleise C, Chudyk J, Ceratto $\mathrm{R}$, et al: Association between hemodynamic conditions and occlusion times after flow diversion in cerebral aneurysms. J Neurointerv Surg 7:286-290, 2015

6. Xiang J, Ma D, Snyder KV, Levy EI, Siddiqui AH, Meng H: Increasing flow diversion for cerebral aneurysm treatment using a single flow diverter. Neurosurgery 75:286-294, 2014 